\title{
ON THE IRREDUCIBILITY OF WEIGHTED SHIFTS
}

\author{
NOBORU SUZUKI
}

Let $\left\{\phi_{n}\right\}(n=0,1, \cdots)$ be an orthonormal basis of a Hilbert space $H$ and let $\left\{\alpha_{n}\right\}$ be a bounded sequence of complex scalars. Then a unilateral weighted shift $A$ with weights $\alpha_{n}$ is the operator on $H$ defined by

$$
A \phi_{n}=\alpha_{n} \phi_{n+1} \quad(n=0,1, \cdots),
$$

and its adjoint is given by

$$
A^{*} \phi_{0}=0 \quad \text { and } \quad A^{*} \phi_{n}=\bar{\alpha}_{n-1} \phi_{n-1} \quad(n=1,2, \cdots) .
$$

It is known that a unilateral shift, more generally, a unilateral weighted shift with nonzero weights is irreducible, i.e., it has no nontrivial reducing subspace. In the present paper we shall show the strong irreducibility of a unilateral weighted shift with nonzero weights in the following sense:

THEOREM 1. Every operator on a Hilbert space which is similar to a unilateral weighted shift with nonzero weights is irreducible.

As a striking application of this result, we shall give a satisfactory answer to the question: which unilateral weighted shifts with nonzero weights are spectral in the sense of Dunford? Indeed, it will be shown that such a weighted shift can be spectral only if it is quasi-nilpotent.

Theorem 2. A unilateral weighted shift with nonzero weights is a spectral operator if and only if it is quasi-nilpotent.

If $A$ is a unilateral weighted shift with weights $\alpha_{n}$, then a straightforward computation shows

$$
\left\|A^{k}\right\|=\sup _{n}\left|\prod_{i=0}^{k-1} \alpha_{n+i}\right| \quad(k=1,2, \cdots),
$$

and hence the spectral radius $\gamma(A)$ is equal to $\lim _{k} \sup _{n}\left|\prod_{i=0}^{k-1} \alpha_{n+i}\right|^{1 / k}$ (cf. [2]). It follows that $A$ is quasi-nilpotent if and only if

$$
\sup _{n}\left|\prod_{i=0}^{k-1} \alpha_{n+i}\right|^{1 / k} \rightarrow 0 \quad \text { as } \quad k \rightarrow \infty
$$

Received by the editors January 10, 1969. 
Now let us consider a nonzero monotone shift $A$ with weights $\alpha_{n}$ (i.e., $\left|\alpha_{n}\right| \leqq\left|\alpha_{n+1}\right|$ for each $n$ ). Stampfli [3] has shown that such an $A$ is not spectral. Obviously this fact may be derived from the above theorem. In fact, let $N$ denote the smallest index such that $\alpha_{N} \neq 0$. Then, having noticed that the restriction of $A$ to the subspace generated by $\phi_{0}, \phi_{1}, \cdots, \phi_{N-1}$ is zero, the subspace $K$ generated by $\phi_{N}, \phi_{N+1}, \cdots$ is a reducing subspace of $A$ and the restriction $A \mid K$ of $A$ to $K$ is a unilateral weighted shift on $K$ with nonzero weights. Thus, by what we have observed above, $A \mid K$ is not quasi-nilpotent, that is, it is not spectral by Theorem 2 . It follows that $A$ itself is not spectral. More generally, a similar argument shows the following

Corollary. Let $A$ be a unilateral weighted shift with weights $\alpha_{n}$. If $\alpha_{0}=\alpha_{1}=\cdots=\alpha_{N}=0$ and there is a positive constant $c$ such that $\left|\alpha_{n}\right| \geqq c$ for all $n>N$, then $A$ is not a spectral operator.

1. In what follows, $A$ always means a unilateral weighted shift with nonzero weights $\alpha_{n}$ associated with an orthonormal basis $\left\{\phi_{n}\right\}$ $(n=0,1, \cdots)$ of a Hilbert space $H$. The proof of Theorem 1 is based on a specific property of the system of invariant subspaces of $A^{*}$.

Lemma. The Hilbert space $H$ can not be expressed as an algebraic direct sum of two nontrivial invariant subspaces of $A^{*}$.

Proof. Suppose that $H$ is decomposed in the algebraic direct sum of nontrivial invariant subspaces $\mathfrak{T}$ and $\Re$ of $A^{*}$. Since $\mathfrak{N} \cap \Re=\{0\}$, we may assume that at least one of the subspaces $\mathscr{T}$ and $\mathscr{N}$ does not contain the vector $\phi_{0}$. Let $\phi_{0} \notin \Re$. Then $\phi_{0}=\psi_{1}+\psi_{2}$, where $\psi_{1} \in \mathbb{M}$ and $\psi_{2} \in \Re$. Consider the Fourier expansions of $\psi_{1}$ and $\psi_{2}$ :

$$
\psi_{1}=\sum_{n} \lambda_{n} \phi_{n}
$$

where $\lambda_{n} \neq 0$ for some $n \geqq 1$ (recall that $\left.\phi_{0} \notin \mathfrak{N}\right)$;

$$
\psi_{2}=\sum_{n} \mu_{n} \phi_{n} .
$$

Then $\phi_{0}=\sum_{n}\left(\lambda_{n}+\mu_{n}\right) \phi_{n}$ implies that $\mu_{0}=1-\lambda_{0}$ and $\mu_{n}=-\lambda_{n}(n \geqq 1)$. Thus $-\psi_{2}=\left(\lambda_{0}-1\right) \phi_{0}+\sum_{n \gtrless 1} \lambda_{n} \phi_{n}$. Since $\Re$ is invariant under $A^{*}$, we have

$$
A^{*}\left(-\psi_{2}\right)=\sum_{n \geqq 1} \lambda_{n} \bar{\alpha}_{n-1} \phi_{n-1} \in \Re .
$$

On the other hand, we have

$$
A^{*} \psi_{1}=\sum_{n \geq 1} \lambda_{n} \bar{\alpha}_{n-1} \phi_{n-1} \in \mathfrak{T} .
$$


Consequently, $\psi \equiv \sum_{n \geq 1} \lambda_{n} \bar{\alpha}_{n-1} \phi_{n-1} \in \Re \cap \Re$. However, since $\alpha_{n} \neq 0$ for all $n$ and $\lambda_{n} \neq 0$ for some $n \geqq 1$, the vector $\psi$ is nonzero. This contradicts $\mathscr{N} \cap \mathscr{N}=\{0\}$.

Proof of Theorem 1. Let $B$ be an operator on $H$ which is similar to $A$. That is, there is an invertible operator $T$ on $H$ such that $B$ $=T A T^{-1}$. Assume that $B$ has a nontrivial reducing subspace $\mathfrak{N}$. Then $\mathfrak{T}$ and its orthogonal complement $\mathfrak{N}^{\perp}$ are invariant under $B^{*}=\left(T^{*}\right)^{-1} A^{*} T^{*}$. Hence $T^{*} \mathscr{N C}$ and $T^{*} \mathfrak{N} \mathcal{C}^{\perp}$ are nontrivial invariant subspaces of $A^{*}$, and further $H$ is decomposed in the direct sum

$$
H=T^{*} \mathfrak{M}+T^{*} \mathfrak{N}^{\perp},
$$

which is impossible by the above lemma.

2. Before proving Theorem 2, let us recall that a spectral operator is the sum $S+N$ of a scalar type operator $S$ and a quasi-nilpotent operator $N$ commuting with $S$, and a scalar type operator on a Hilbert space is similar to a normal operator (see [1]). The proof is completed by the same argument as that employed in [4].

Proof of Theorem 2. We need only to prove that if the weighted shift $A$ is spectral, then it is quasi-nilpotent. In this case, as we have mentioned above, $A$ is similar to a spectral operator $\tilde{A}$ whose scalar part $\tilde{S}$ is normal. By Theorem $1, \tilde{A}$ is irreducible, that is to say, the von Neumann algebra generated by $\tilde{A}$ is the algebra $\mathscr{L}(H)$ of all operators on $H$. Since $\tilde{S}$ commutes with $\tilde{A}$, the normality of $\tilde{S}$ implies that $\tilde{S}^{*}$ also commutes with $\tilde{A}$. Thus $\tilde{S}$ belongs to the center of $\mathscr{L}(H)$ and hence $\tilde{S}$ must be a scalar multiple $\lambda I$ of the identity operator $I$. This implies that $A=\lambda I+N$, where $N$ is quasi-nilpotent. It follows that the spectrum $\sigma(A)$ of $A$ consists of only one point $\lambda$. But $\sigma(A)$ is circular symmetry, i.e., if $|\alpha|=1$, then $\alpha \lambda \in \sigma(A)$ (see [2: Problem 75]). Thus $\lambda$ must be 0 .

Added In Proof. H. Behncke has proved independently Theorem 1 in his manuscript, $A$ class of irreducible operators, but his proof is entirely different from ours.

\section{REFERENCES}

1. N. Dunford, A survey of the theory of spectral operators, Bull. Amer. Math. Soc. 64 (1958), 217-274.

2. P. R. Halmos, A Hilbert space problem book, Van Nostrand, Princeton, N. J.. 1967.

3. J. G. Stampfli, Which weighted shifts are subnormal, Pacific J. Math. 17 (1966), 367-379.

4. N. Suzuki, The structure of spectral operators with completely continuous imagi nary part, Proc. Amer. Math. Soc. 22 (1969), 82-84.

University of California, Irvine 